\title{
Erythematous Plaque on the Scalp With Alopecia
}

Yu-Qing Hu, MD; Xue-Yan Yao, MD; Cheng Zhou, MD; Jian-Zhong Zhang, MD; Yan Zhao, MD

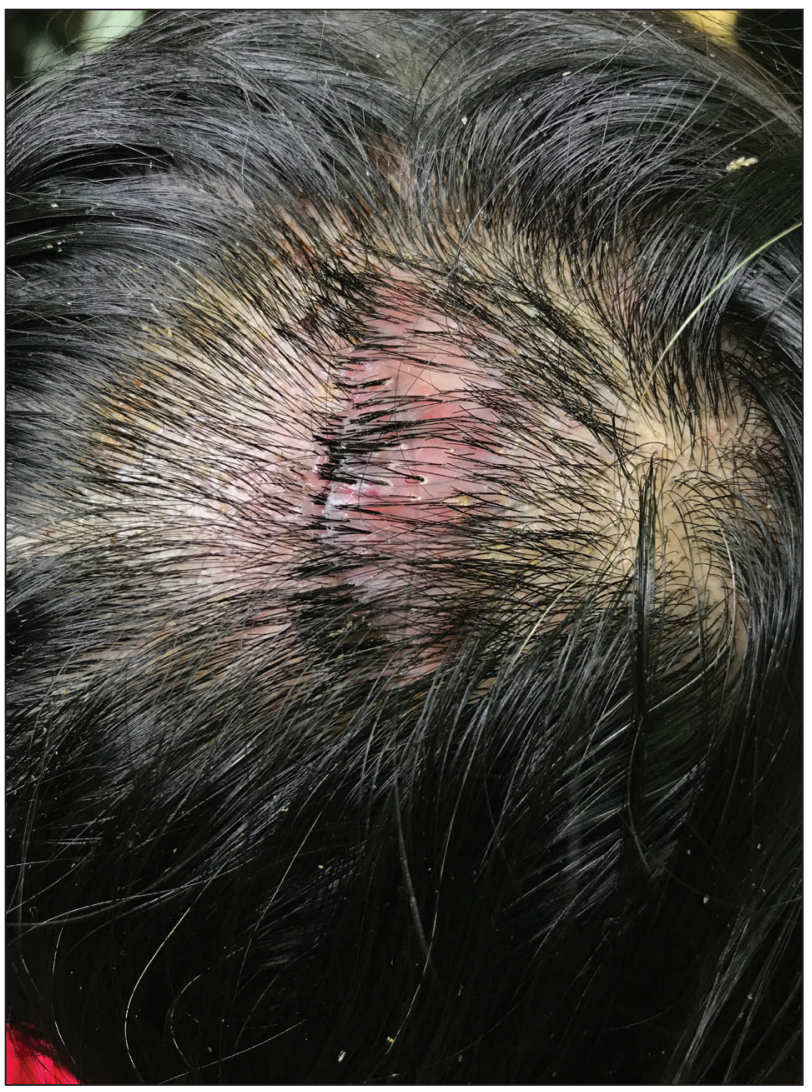

A 37 -year-old woman presented with a $2 \times 6-\mathrm{cm}$, firm, erythematous plaque on the parietal region of the scalp of 1 year's duration. No history of injury to the scalp was noted. The patient noticed hair loss in the affected area in the month prior to presentation. She was afebrile and otherwise asymptomatic. She denied a family history of similar scalp disorders.

\section{WHAT'S THE DIAGNOSIS?}
a. discoid lupus erythematosus
b. dissecting cellulitis of the scalp
c. follicular lichen planus
d. folliculitis keloidalis nuchae
e. tufted hair folliculitis 


\section{THE DIAGNOSIS:}

\section{Tufted Hair Folliculitis}

( ermoscopic examination revealed multiple hair tufts of 5 to 20 normal hairs emerging from single dilated follicular openings (Figure 1). The density of hair follicles was reduced with adherent yellowwhite scales that encircled the dilated follicular orifices. Histopathology revealed hyperkeratosis and parakeratosis in the stratum corneum. Infiltration of lymphocytes, neutrophils, plasma cells, and eosinophils around the upper portions of the follicles also was found. Multiple hairs emerging from a single dilated follicular ostia with prominent fibrosis of the dermis were seen (Figure 2). Based on the clinical and histopathological findings, the patient was diagnosed with tufted hair folliculitis (THF). She was treated with minocycline $100 \mathrm{mg}$ once daily and an intralesional betamethasone injection $5 \mathrm{mg}$ once daily. After 2 weeks of treatment, the lesion improved and decreased in size to $1 \times 1 \mathrm{~cm}$ in diameter; however, the hair tufts and scarring alopecia remained.

Tufted hair folliculitis is a rare inflammatory condition of the scalp characterized by a peculiar tufting of hair that was first described by Smith and Sanderson ${ }^{1}$ in 1978. Most patients present with a patch or plaque on the parietal or occipital region of the scalp. The condition may lead to the destruction of follicular units, resulting in permanent scarring alopecia. ${ }^{2}$ Histopathology in our patient revealed perifollicular inflammation, and several follicles could be seen converging toward a common follicular duct with a widely dilated opening, consistent with the diagnosis of THF.

The pathogenic mechanisms of THF are unclear. Primary hair tufting, local trauma, tinea capitis, nevoid

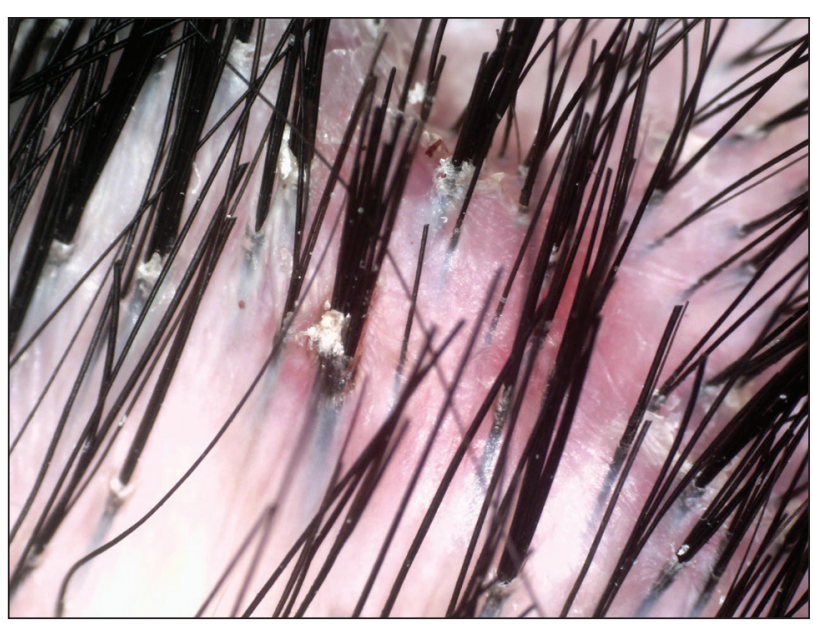

FIGURE 1. Tufted hair folliculitis. Dermoscopic examination showed an erythematous plaque and multiple hair tufts composed of 5 to 20 normal hair shafts emerging from single dilated follicular openings (original magnification $\times 40$ ). malformation, and Staphylococcus aureus infection have been proposed as causative pathomechanisms. ${ }^{3}$ Typically there is no history of underlying disease or trauma on the scalp; however, secondary changes may have occurred following unrecognized trauma or repeated stimuli. Staphylococcal infections may play a notable role in inducing THF. Ekmekci and Koslu ${ }^{4}$ reported that a local inflammatory process led to the destruction of adjacent follicles, which subsequently amalgamated to form a common follicular duct due to local fibrosis and scarring. However, Powell et $\mathrm{al}^{5}$ found no evidence of local immune suppression or immune failure that could explain the abnormal host response to a certain
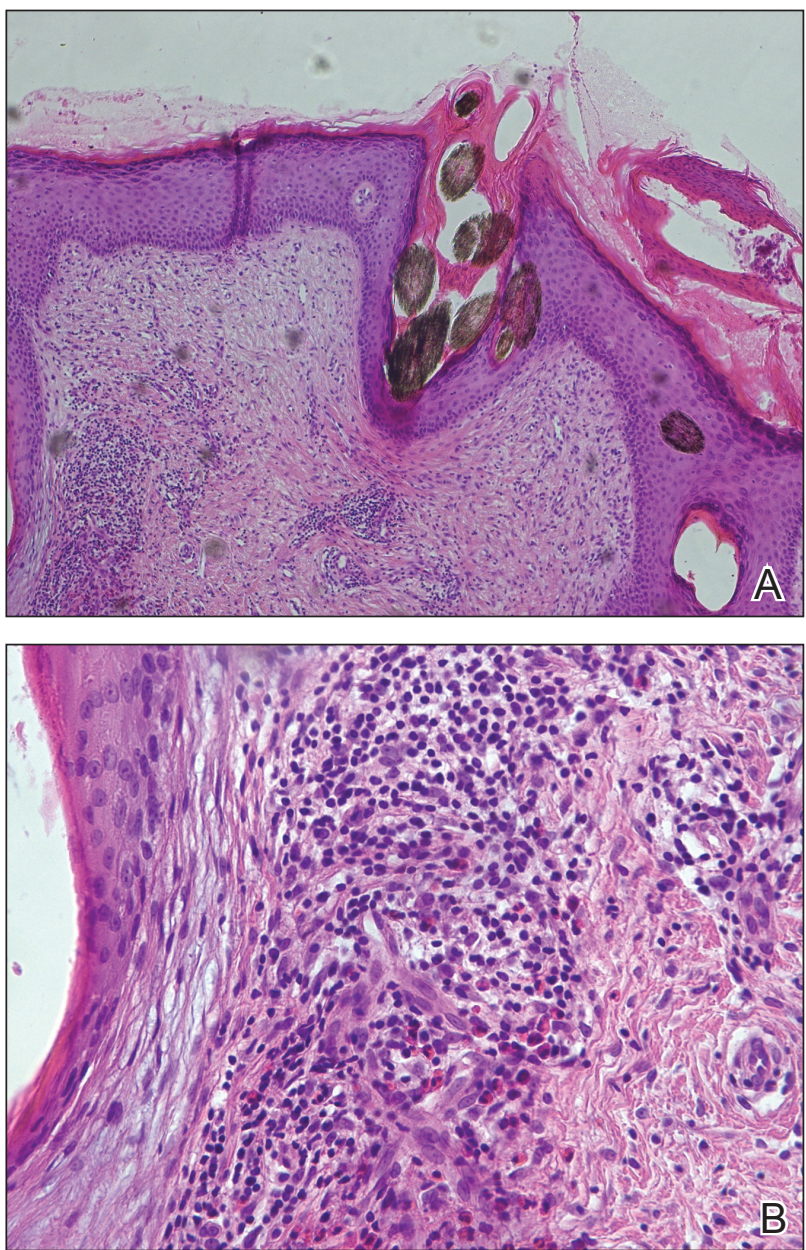

FIGURE 2. Tufted hair folliculitis. A, Histologic examination revealed multiple normal hairs emerging from single dilated hair follicles (H\&E, original magnification $\times 200$ ). B, A mixed inflammatory infiltrate composed of lymphocytes, neutrophils, plasma cells, and eosinophils around the upper portions of the follicles was seen (H\&E, original magnification $\times 800$ ). 
presumptive superantigen. In our patient, the inflammatory injury was mild, and no purulent exudation was found from the dilated follicular openings. Because the patient had applied an antibiotic ointment prior to presentation, bacterial cultures from biopsy specimens were not appropriate.

The differential diagnosis of THF includes folliculitis decalvans, folliculitis keloidalis nuchae, dissecting cellulitis of the scalp, and follicular lichen planus. ${ }^{6}$ In our patient, folliculitis keloidalis nuchae and dissecting cellulitis of the scalp were excluded because no keloid or purulent inflammation was found. The diagnosis of follicular lichen planus was not taken into consideration because characteristic pathology such as liquefaction degeneration of basal cells was not observed. Folliculitis decalvans was considered to be a possible cause of the alopecia in our patient. It also was suggested that hair tufting could be a secondary phenomenon, occurring in several inflammatory disorders of the scalp. Powell et $\mathrm{al}^{5}$ concluded that THF should be considered as a distinctive clinicohistologic variant of folliculitis decalvans characterized by multiple hair tufts with patches of scarring alopecia. This hypothesis corresponded with our patient's clinical manifestation and histopathology.

Conventional treatment of THF includes topical antiseptics and oral antibiotics (eg, flucloxacillin, erythromycin, tetracycline, doxycycline), but reduction in hair bundling rarely has been observed after antibiotic treatment. Although good prognosis has been reported after surgical excision of the involved areas, it can only be performed in small lesions. ${ }^{6}$ Pranteda et $\mathrm{al}^{7}$ reported that combination therapy with oral rifampin and oral clindamycin can prevent relapse longterm. Combination therapy for 10 weeks also was effective in 10 of 18 patients with THF. ${ }^{5}$ Rifampin is an effective therapeutic modality to control the progression of THF as well as prevent relapse; however, long-term use should be avoided to prevent hepatic or renal side effects. ${ }^{7}$ Our patient was successfully treated with intralesional betamethasone and oral minocycline to reduce the inflammation and prevent the expansion of scarring alopecia.

Acknowledgment-The authors thank Xue Chen, MD (Beijing, China), for writing support.

\section{REFERENCES}

1. Smith NP, Sanderson KV. Tufted folliculitis of the scalp. J R Soc Med. 1978;71:606-608.

2. Broshtilova V, Bardarov E, Kazandjieva J, et al. Tufted hair folliculitis: a case report and literature review. Acta Dermatovenerol Alp Pannonica Adriat. 2011;20:27-29.

3. Gungor S, Yuksel T, Topal I. Tufted hair folliculitis associated with Melkersson-Rosenthal syndrome and hidradenitis suppurativa. Indian J Dermatol Venereol Leprol. 2014;80:484-487.

4. Ekmekci TR, Koslu A. Tufted hair folliculitis causing skullcap -pattern cicatricial alopecia. J Eur Acad Dermatol Venereol. 2006;20:227-229.

5. Powell JJ, Dawber RP, Gatter K. Folliculitis decalvans including tufted folliculitis: clinical, histological and therapeutic findings. Br J Dermatol. 1999;140:328-333.

6. Baroni A, Romano F. Tufted hair folliculitis in a patient affected by pachydermoperiostosis: case report and videodermoscopic features. Skinmed. 2011;9:186-188.

7. Pranteda G, Grimaldi M, Palese E, et al. Tufted hair folliculitis: complete enduring response after treatment with rifampicin. J Dermatolog Treat. 2004;15:396-398.

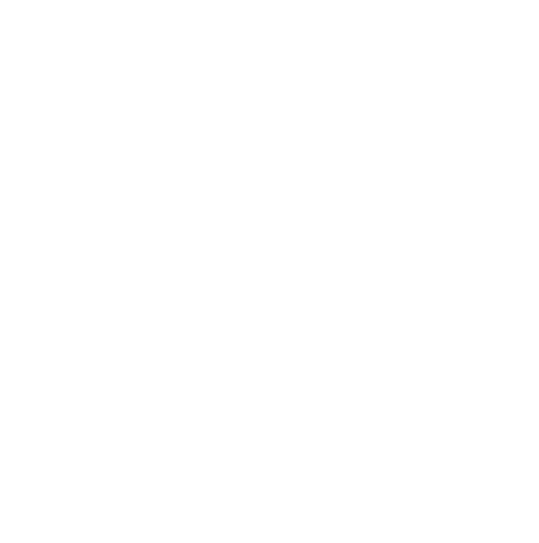

